\title{
Article
}

\section{Suxiao Jiuxin pill promotes exosome secretion from mouse cardiac mesenchymal stem cells in vitro}

\author{
Xiao-fen RUAN ${ }^{1,3}$, Cheng-wei JU ${ }^{2,3}$, Yan SHEN ${ }^{3}$, Yu-tao LIU $^{3}$, II-man $\mathrm{KIM}^{3}$, Hong YU ${ }^{4}$, Neal WEINTRAUB ${ }^{3}$, Xiao-long WANG ${ }^{1, *}$, \\ Yaoliang TANG ${ }^{3, *}$ \\ ${ }^{1}$ Cardiovascular Department, Cardiovascular Research Institute of Traditional Chinese Medicine, Shuguang Hospital of Shanghai \\ University of Traditional Chinese Medicine, Shanghai 201203, China; ${ }^{2}$ Department of Cardiology, Zhongda Hospital, Medical School \\ of Southeast University, Nanjing 210009, China; ${ }^{3}$ Medical College of Georgia, Augusta University, Augusta, GA, USA; ${ }^{4}$ Department of \\ Cardiology, Second Affiliated Hospital, College of Medicine, Zhejiang University, Hangzhou 310009, China
}

\begin{abstract}
Cardiac mesenchymal stem cells (C-MSCs) are endogenous cardiac stromal cells that play a role in heart repair after injury. C-MSCderived exosomes (Exo) have shown protective effects against apoptosis induced by acute myocardial ischemia/reperfusion. Suxiao Jiuxin pill (SJP) is a traditional Chinese medicine (TCM) formula used in China for the treatment of acute myocardial ischemia, which contains tetramethylpyrazine (TMP) and borneol (BOR) as major components. In this study, we investigated whether SJP treatment affected exosome release from C-MSCs in vitro. C-MSCs prepared from mice were treated with SJP $(62.5 \mu \mathrm{g} / \mathrm{mL}), \mathrm{TMP}(25 \mu \mathrm{g} / \mathrm{mL})$ or BOR $(15 \mu \mathrm{g} / \mathrm{mL})$. Using an acetylcholinesterase activity assay, we found that both SJP and TMP treatment significantly increased exosome secretion compared to the control ethanol treatment. The neutral sphingomyelinase 2 (nSMase2) pathway was important in exosome formation and packaging. But neither the level of nSMase2 mRNA nor the level of protein changed following SJP, TMP or BOR treatment, suggesting that SJP stimulated exosome release via an nSMase2-independent pathway. The Rab27a and Rab27b GTPases controlled different steps of the exosome secretion pathway. We showed that SJP treatment significantly increased the protein levels of Rab27a, SYTL4 (Rab27a effector) and Rab27b compared with the control treatment. SJP treatment also significantly upregulated the mRNA level of Rab27b, rather than Rab27a. Moreover, SJP-induced increase of C-MSC-exosome release was inhibited by Rab27b knockdown, suggesting that SJP promotes exosome secretion from C-MSCs via a GTPase-dependent pathway. This study reveals a novel mechanism for SJP in modulating cardiac homeostasis.
\end{abstract}

Keywords: acute myocardial ischemia; cardiac mesenchymal stem cells; exosomes; Rab27; GTPase; nSMase2; traditional Chinese medicine; Suxiao Jiuxin pill; tetramethylpyrazine; borneol

Acta Pharmacologica Sinica (2018) 39: 569-578; doi: 10.1038/aps.2018.19; published online 15 Mar 2018

\section{Introduction}

The Suxiao Jiuxin pill (SJP) is a traditional Chinese herbal medicine (TCM) that has a clinical application in angina pectoris with notable cardioprotective effects ${ }^{[1-4]}$. The major components of SJP include tetramethylpyrazine (TMP) and borneol (BOR). Tetramethylpyrazine is the rhizome of Ligusticum chuanxiong Hort ${ }^{[5]}$. The mechanisms of cardioprotection by SJP are not well understood. Li et al ${ }^{[6]}$ reported that SJP plays an important role in anti-inflammation by down-regulating the expression of Peroxisome proliferator-activated receptor gamma (PPARү) and nuclear factor-kappa beta (NF-kB) pro-

\footnotetext{
${ }^{*}$ To whom correspondence should be addressed.

E-mail yaotang@augusta.edu (Yaoliang TANG); wxlqy0214@163.com (Xiao-long WANG)

Received 2017-08-24 Accepted 2018-01-07
}

teins and inhibiting oxidative stress by upregulating superoxide dismutase (SOD) activity in a high fat diet rat model. Bai et $a l^{[7]}$ demonstrated that SJP has a potent inhibitory effort on vessel constriction induced by $\mathrm{KCl}$ and $\mathrm{U} 46619$ in human internal mammary arteries.

There are resident cardiac mesenchymal stromal cells (C-MSCs) in adult hearts that express the cardiac transcription factor GATA-4 compared with bone marrow stromal cells ${ }^{[8]}$. C-MSCs have cardiac repair properties via paracrine effects that are mediated by either angiogenic factors ${ }^{[9,10]}$ or exosomes $^{[11-13]}$. C-MSC-derived exosomes (Exo) are 40-100 nm in diameter vesicles with a distinct GATA4-responsive miR-451 enrichment ${ }^{[13]}$. We have reported that Exo protect cardiomyocytes from acute myocardial ischemia/reperfusion injury ${ }^{[13]}$.

Exosomes secreted from stem cells can be taken up by their surrounding tissues. Therefore, protein, RNA, and lipids from 
stem cell-derived exosomes can enter into neighboring cells, such as cardiomyocytes, and modulate their cell signaling. The neutral sphingomyelinase2 (nSMase2) pathway and the small GTPase Rab27 are two important signaling pathways that control exosome generation and secretion. Inhibition of the nSMase2 pathway using GW4869, a pharmacological compound, effectively inhibits exosome biogenesis ${ }^{[14]}$. In additional, Guo et al ${ }^{[15]}$ reported that knockdown of nSMase2 in mouse cells using shRNA also decreases the release of exosomes. The small GTPases Rab27a and Rab27b, members of the small GTPase Rab family, can control membrane trafficking and microvesicle transport, in particular the secretion of exosomes. Zhang et al ${ }^{[16]}$ confirmed that inhibition of Rab27 results in decreased exosome release into the culture medium of breast cancer cells. It remains unknown whether SJP treatment impacts C-MSC function regarding exosome secretion and related mechanisms. In this study, we investigated the effect of SJP on exosome secretion in C-MSCs in vitro and associated signaling pathways. We found that SJP treatment increases exosome release from C-MSCs with a significant upregulation of the Rab27a, SYTL4 (Rab27a effector), and Rab27b proteins, as well as the small GTPases, and was independent of the nSMase2 pathway.

\section{Materials and methods C-MSC isolation and culture}

Cardiac mesenchymal stem cells were isolated from the hearts of 2- to 3-month-old male C57BL/ 6 mice (The Jackson Laboratory, Bar Harbor, ME, USA) via a 2-step procedure as described previously ${ }^{[8,17]}$. Briefly, in step 1 , ventricular cardiac tissues were minced into $1 \mathrm{~mm}^{3}$ size, digested with $0.1 \%$ collagenase IV and $1 \mathrm{U} / \mathrm{mL}$ dispase in DMEM/F-12 and explanted in fibronectin/gelatin coated wells $(0.5 \mathrm{mg}$ fibronectin in 100 $\mathrm{mL} 0.1 \%$ gelatin). Culture cardiac explants were maintained until the small round phase-bright cells migrated from the adherent explants and proliferated over a fibroblast layer. In step 2, Sca-1+ cells were enriched from the phase-bright cells through a mouse hematopoietic lineage-depletion cocktail kit (STEMCELL Technologies, Vancouver, Canada), followed by enrichment for Sca-1+ cells via magnetic-activated cell sorting (MACS) with Sca-1 magnetic beads (Miltenyi Biotec Inc, Auburn, CA, USA) according to the manufacturers' protocols. The selected Sca-1 cells were cultured and maintained in complete DMEM/F12 media containing 10\% fetal bovine serum, $200 \mathrm{mmol} / \mathrm{L}$ L-glutamine, $55 \mathrm{nmol} / \mathrm{L} \beta$-mercaptoethanol, and $1 \%$ MEM nonessential amino acids.

\section{Flow cytometry}

Flow cytometry analyses of cultured C-MSCs were performed with a BD LSRII flow cytometer and BD FACSDiva ${ }^{\mathrm{TM}}$ software as previously described ${ }^{[17]}$. Briefly, C-MSCs were blocked with $5 \%$ rat serum and stained with a panel of conjugated antibodies, including anti-Sca-1-PE (BD Biosciences, San Jose, CA, USA), anti-CD105-APC (BioLegend, San Diego, CA, USA), anti-c-kit-FITC (BD Biosciences), and anti-CD45-Alexa 700 (BD Biosciences), or isotype-matched control antibodies (eBiosci- ence, Waltham, MA, USA).

\section{Drug preparation, cell treatment and exosome purification}

The origin, medicinal composites, and processing technology of SJP (Tianjin Zhongxin Pharmaceutical Group Co, Ltd, Tianjin, China) were strictly standardized on the basis of marker compounds to achieve quality control according to the Chinese Pharmacopoeia $2015^{[18]}$. The dosages of SJP and BOR for cell culture are based on therapeutic drug concentrations in plasma from patients who received effective SJP treatment ${ }^{[4,19]}$, and the dosage of TMP for cell culture is based on a previous experimental report ${ }^{[20]}$. In cell culture experiments, SJP was diluted in ethanol to $62.5 \mathrm{mg} / \mathrm{mL}$ (1000× stock), TMP was diluted in ethanol to $25 \mathrm{mg} / \mathrm{mL}$ (1000× stock), and BOR was diluted in ethanol to $15 \mathrm{mg} / \mathrm{mL}$ (1000× stock). C-MSCs in 15-cm dishes were treated with $0.1 \%$ ethanol (Ctrl), $62.5 \mu \mathrm{g} / \mathrm{mL} \mathrm{SJP}{ }^{[4]}, 25$ $\mu \mathrm{g} / \mathrm{mL} \mathrm{TMP}^{[20]}$ or $15 \mu \mathrm{g} / \mathrm{mL} \mathrm{BOR}^{[19]}$ in culture medium with 10\% exosome-depleted FBS for $48 \mathrm{~h}$. The C-MSC-released exosomes were purified as described in the literature with modification $^{[21]}$. Briefly, supernatants were centrifuged at $150 \times g$ for 10 min to eliminate cells, followed by filtration through a $0.22 \mu \mathrm{m}$ filter to remove cell debris. Exosomes in medium were precipitated with $5 \times$ polyethylene glycol 4000 (PEG4000, 8.5\% final concentration) with $10 \times \mathrm{NaCl}(0.4 \mathrm{~mol} / \mathrm{L}$ final concentration) overnight at $4{ }^{\circ} \mathrm{C}$, followed by centrifugation at $2100 \times g$ for 30 min. The pellets were resuspended in PBS and stored at $-80^{\circ} \mathrm{C}$ for future use.

\section{Immuno-electron microscopy imaging}

Standard immuno-electron staining was performed with antiCD63 as described previously ${ }^{[22]}$. Fixed exosome preparations were placed on carbon-Formvar coated 200 mesh nickel grids for $30 \mathrm{~min}$. The grids were then quenched with $1 \mathrm{~mol} / \mathrm{L}$ ammonium chloride for $30 \mathrm{~min}$ and blocked with $0.4 \%$ BSA in PBS for $2 \mathrm{~h}$. The grids were washed with PBS three times and then incubated with primary rabbit-anti-CD63 (1:100, Santa Cruz Biotechnology, Inc, Santa Cruz, CA, USA) for $1 \mathrm{~h}$. Then, the grids were washed with $\mathrm{ddH}_{2} \mathrm{O}$ and PBS and floated on drops of $1.4 \mathrm{~nm}$ anti-rabbit nanogold (1:1000, Nanoprobes, Inc) in blocking buffer for $1 \mathrm{~h}$. After enhancement with HQ Silver (gold enhancement reagent, Nanoprobes, Inc), the samples were wicked dry and then allowed to air dry before observation in a transmission electron microscope (JEOL JEM 1230, Peabody, MA, USA). TEM sample preparation and imaging was performed at the Electron Microscopy and Histology Core Laboratory at Augusta University (www.augusta.edu/mcg/ cba/emhisto/).

\section{Zeta analysis}

We measured the exosome particle size with nanoparticle tracking analysis (NTA) using ZetaView PMX 110 (Particle Metrix, Meerbusch, Germany) and the corresponding software ZetaView 8.02.28. Isolated exosome samples were appropriately diluted using $1 \times$ PBS buffer (Life Technologies, Carlsbad, CA, USA) to measure the particle size. NTA measurement was recorded and analyzed at 11 positions. The ZetaView sys- 
tem was calibrated using $100 \mathrm{~nm}$ polystyrene particles. Temperature was maintained at approximately $25^{\circ} \mathrm{C}$.

\section{Small-interference RNA (siRNA) transfection}

Silencer ${ }^{\mathrm{TM}}$ Select Pre-Designed siRNA for mouse Rab27a (Rab27a-siRNA, sirna_id: s62663, Thermo Fisher Scientific, Waltham, MA, USA), mouse Rab27b (Rab27b-siRNA, sirna_id: s95999, Thermo Fisher Scientific) and a non-targeting control siRNA (NT-siRNA, sc-37007, Santa Cruz Biotechnology, Inc, Dallas, TX, USA) were used for knockdown experiments. For transfection, $100 \mathrm{nmol} / \mathrm{L}$ Rab27a-siRNA, Rab27b-siRNA, or NT-siRNA was transfected into C-MSCs in $100 \mu \mathrm{L}$ "R buffer" using the Neon electroporation transfection system (Invitrogen) operating at $1350 \mathrm{~V}$ with two $30 \mathrm{~ms}$ pulses, and then the cells were incubated for an additional $48 \mathrm{~h}$ before treatment.

\section{Assessment of acetylcholinesterase (AChE) activity}

Acetylcholinesterase activity was used to determine exosome release as described previously ${ }^{[23]}$. We use the Amplex ${ }^{\circledR}$ Red Acetylcholine/Acetylcholinesterase Assay Kit (Thermo Fisher) for the acetylcholinesterase assay. Briefly, $10 \mu \mathrm{L}$ exosomal fractions were suspended in $90 \mu \mathrm{L}$ of PBS. Then, $100 \mu \mathrm{L}$ of this PBS-diluted exosome fraction was added to individual wells of a 96-well flat-bottomed microplate. Next, $100 \mu \mathrm{L}$ of the Amplex Red reagent/HRP/choline oxidase/acetylcholine working solution was added to exosome fractions in a final volume of $200 \mu \mathrm{L}$. After $30 \mathrm{~min}$ of incubation at room temperature in the dark, the fluorescence was measured in a fluorescence microplate reader using emission detection at $590 \mathrm{~nm}$. For each well, the value is corrected via subtracting the background fluorescence from the no acetylcholinesterase control and then normalized to the protein concentration of treated C-MSCs.

\section{Isolation and quantification of messenger RNA}

Total RNA from C-MSCs was extracted by RNAzol RT (Molecular Research Center, Inc, Cincinnati, OH, USA) following the manufacturer's instructions. cDNA was synthesized from total RNA using the RevertAid First Strand cDNA Synthesis kit (Thermo Scientific). The cDNA was used to perform quantitative PCR on a CFX96 Touch Real-Time PCR Detection System (Bio-Rad Laboratories, Hercules, CA, USA) using PowerUp SYBR Green Master Mix (Thermo Fisher). Amplification was performed at $50^{\circ} \mathrm{C}$ for $2 \mathrm{~min}, 95^{\circ} \mathrm{C}$ for $2 \mathrm{~min}$, followed by 40 cycles of $95^{\circ} \mathrm{C}$ for $15 \mathrm{~s}$, and $60^{\circ} \mathrm{C}$ for 1 min with the primers listed in Table 1.

\section{Western blotting assay}

Purified exosomes or treated C-MSCs were assessed for their protein content using a BCA Protein Assay Kit (Pierce, Rockford, IL, USA) and then resolved on a $10 \%$ sodium dodecyl sulfate Bis-tris gel, and transferred to an Odyssey ${ }^{\circledR}$ Nitrocellulose membrane (LI-COR Biosciences, Lincoln, NE, USA). For the Odyssey technology, the membrane was blocked with Odyssey blocking buffer (LICOR Biosciences) and probed with rabbit anti-CD63 (1:250, Santa Cruz Biotechnology, Inc), mouse
Table 1. Prime list.

\begin{tabular}{ll}
\hline \multicolumn{1}{c}{ Gene list } & \multicolumn{1}{c}{ Sequence $\left(5^{\prime}-3^{\prime}\right)$} \\
\hline Rab27a FWD & CAGGAGAGGTTTCGTAGCTTAAC \\
Rab27a REV & GGCTTATCCAGTTTCGGACAT \\
Rab27b FWD & CTTACCACTGCCTTCTTCAGAG \\
Rab27b REV & TGCAGTTGACTCATCCAGTTT \\
SMPD3/nSMase2 FWD & CTACATCGATTCTCCCACCAAC \\
SMPD3/nSMase2 REV & CACAGAGGCTGTCCTCTTAATG \\
GAPDH FWD & TGACATCAAGAAGGTGGGAAG \\
GAPDH REV & AGTGGGAGTTGCTGTTGAAG \\
\hline
\end{tabular}

anti-Tsg101 (1:1000, Thermo Scientific), mouse anti-nSMase2 (1:250, Santa Cruz Biotechnology, Inc), rabbit anti-Rab27a (1:1000, Abcam, UK), rabbit anti-Rab27b (1:1000, Merck Millipore, Burlington, MA, USA), rabbit anti-Sytl4 (1:1000, Abcam), and mouse anti-GAPDH (1:5000, Millipore) at $4{ }^{\circ} \mathrm{C}$ overnight. Then, the membranes were incubated for $1 \mathrm{~h}$ at room temperature with IRDye 680 goat anti-rabbit IgG at 1:10 000 or IRDye 800 goat anti-mouse IgG at 1:10 000 (LI-COR Biosciences). Probed blots were scanned using an Odyssey infrared imager.

\section{Immunofluorescent staining}

For cell staining, C-MSCs plated on 8-well chamber slides (Millipore, Billerica, MA, USA) were treated with drug for $48 \mathrm{~h}$ and fixed with $4 \%$ paraformaldehyde. After blocking with 5\% goat serum, the cells were incubated with rabbit anti-Rab27a (1:200, Abcam) or rabbit anti-Rab27b (1:200; Millipore) at $4{ }^{\circ} \mathrm{C}$ overnight. Primary antibodies were resolved via secondary staining with a goat anti-rabbit Alexa Fluor 488-conjugated antibody (1:400, Life Technologies, Carlsbad, CA, USA). The slides were mounted using VECTASHIELD HardSet Mounting Medium with DAPI (Vector Laboratories, Burlingame, CA, USA).

\section{Statistical analysis}

All values are expressed as the mean \pm standard error of the mean (SEM). Student's $t$-test was used to compare two groups. One-way ANOVA was used for comparisons between 3 or more means. A value of $P<0.05$ indicated statistically significant differences.

\section{Results}

\section{Characterization of C-MSCs}

C-MSCs were obtained using a two-step procedure: cardiacderived cells were grown from enzymatically digested minced adult mouse heart and expanded, and then the C-MSCs were isolated using a hematopoietic lineage-depletion cocktail followed by enrichment for Sca-1+ cells via MACS sorting (Figure 1A). Immunofluorescent staining showed that C-MSCs express GATA4, an early cardiac transcription factor (Figure 1B). The identification of MSCs is currently based on the presence of surface markers (Sca-1 and CD105) and the absence of hematopoietic (c-kit and CD45) antigens ${ }^{[24]}$. Surface marker expression was profiled by flow cytometry. Over $90 \%$ of the 

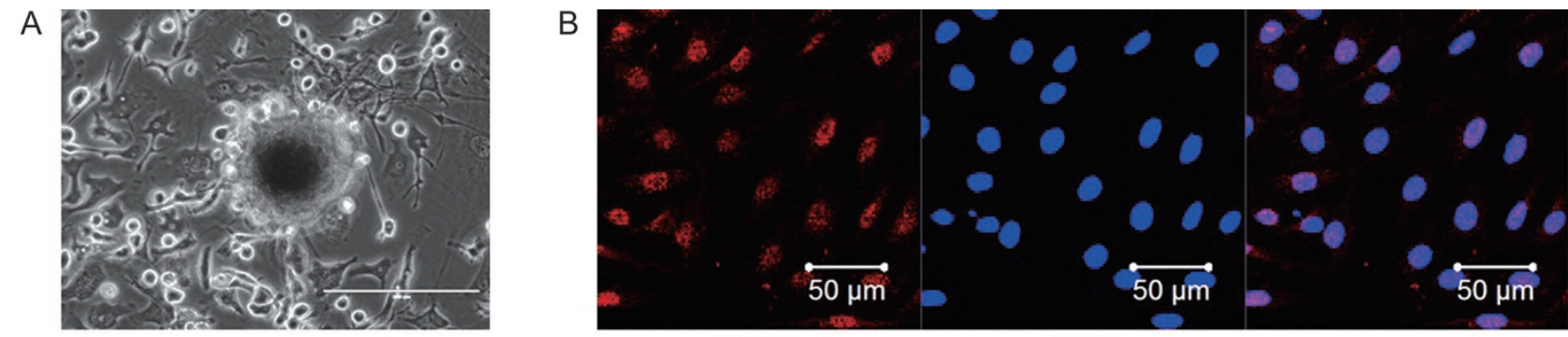

GATA4

DAPI

Merged
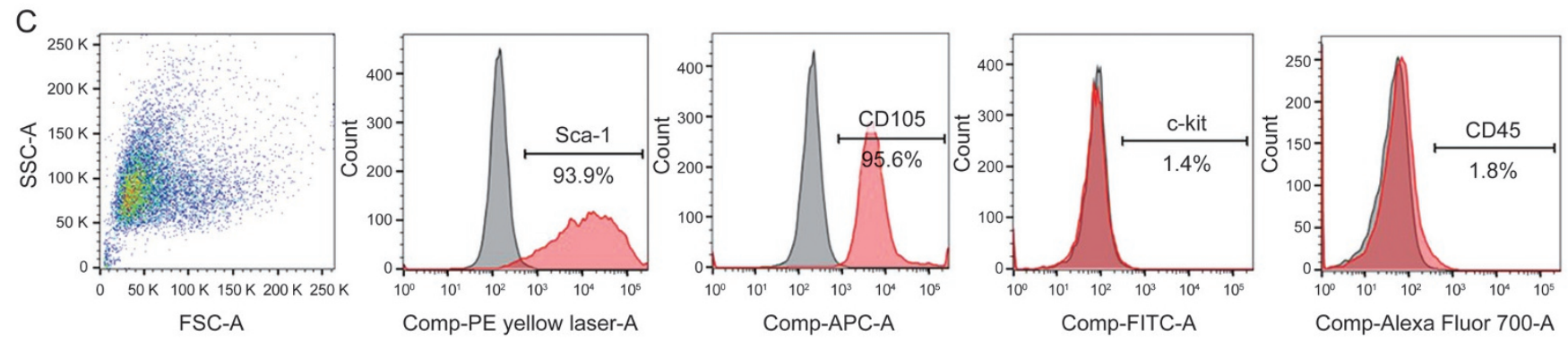

Figure 1. Phenotypic characterization of C-MSCs. (A) Cultured C-MSCs. (B) Immunofluorescent staining of C-MSCs for the cardiac transcription factor GATA4 (red); cell nuclei were counterstained with DAPI (blue). (C) Flow cytometric analyses of C-MSCs for expression of the cell surface markers Sca-1, CD105, c-kit and CD45.

sorted cells were positive for both Sca-1 and CD105 while only approximately $1.4 \%-1.8 \%$ of sorted cells were positive for c-kit and CD45 (Figure 1C). These data indicate that C-MSCs represent a subpopulation of cardiac-derived mesenchymal stem cells.

\section{Characterization of the C-MSC-derived exosomes}

Morphological analysis of the C-MSC-derived exosomes using electron micrograph analysis demonstrated grape-like clusters of vesicles positive for the exosomal marker CD63 (Figure 2A). Western blot analysis of C-MSC-derived exosomes revealed the presence of exosome markers: lysosomal-associated membrane protein 3 (CD63) and ESCRT-I complex subunit
TSG101(Tsg101) (Figure 2B). The size of pelleted structures was determined with dynamic light scattering (DLS) using a ZetaView $^{\circledR}$, a nanoparticle tracking analyzer for hydrodynamic particle size. The pellets consisted of particles with an average size of approximately $100 \mathrm{~nm}$ in diameter, consistent with the characteristic size range of exosomes (Figure 2C).

\section{Effects of SJP on exosome secretion in C-MSCs via AChE activity assay} To determine the effect of SJP on exosome release, we measured exosome release in C-MSCs treated with Ctrl, SJP, TMP and BOR; the latter two are major components of SJP. We performed exosome quantification using an acetylcholine esterase (AChE) activity assay. The AChE enzyme is highly enriched
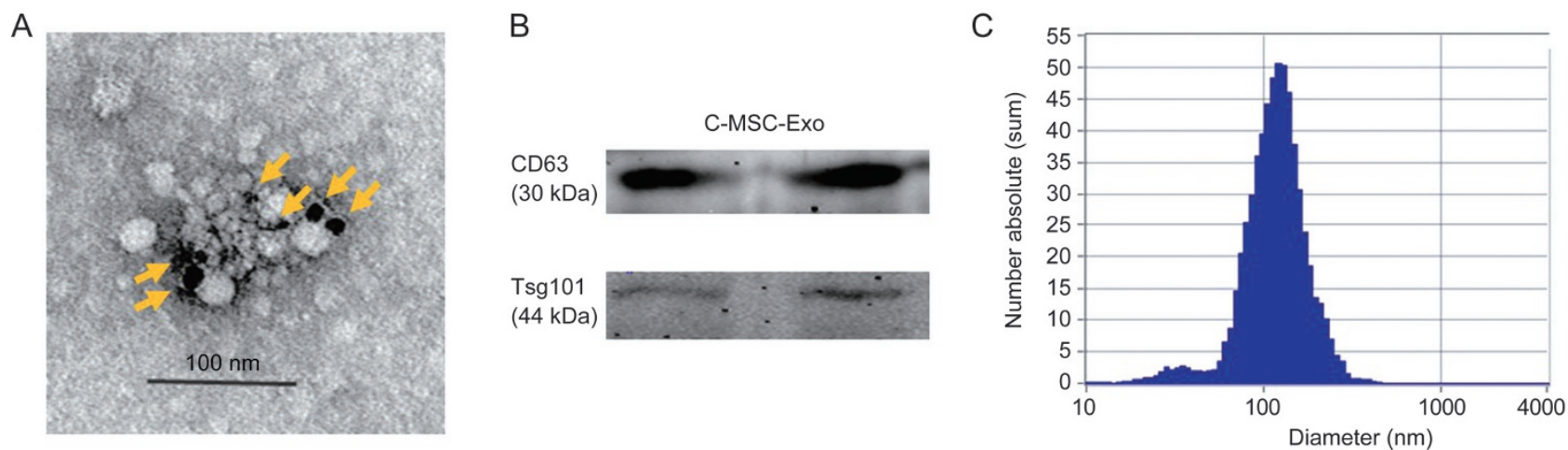

Figure 2. Characterization of C-MSC-derived exosomes. (A) Transmission electron micrograph image of C-MSC-derived exosomes after immunoelectron labeling with an anti-CD63 antibody. Scale bar=100 nm. (B) Western blot results demonstrate the expression of CD63 and Tsg101 in exosomes derived from C-MSCs. (C) Particle size distribution in purified pellets consistent with the size range of exosomes (average size $100 \mathrm{~nm}$ ), measured by ZetaView ${ }^{\circledR}$ Particle Tracking Analyzer. 
A

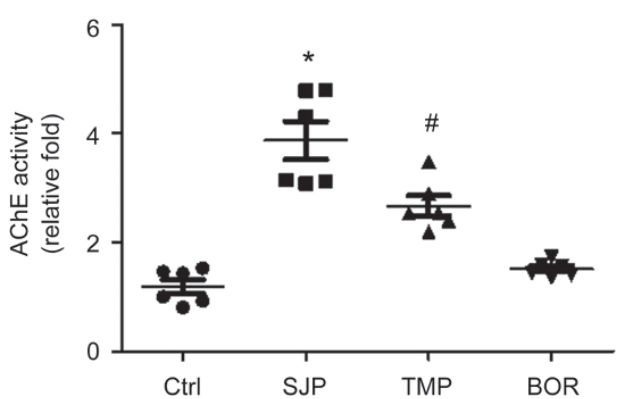

C

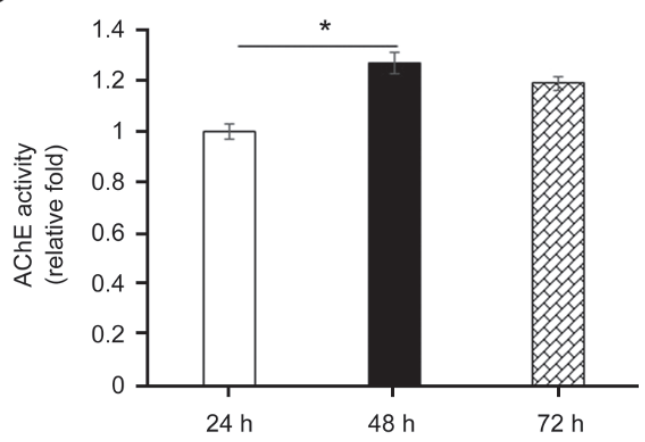

B

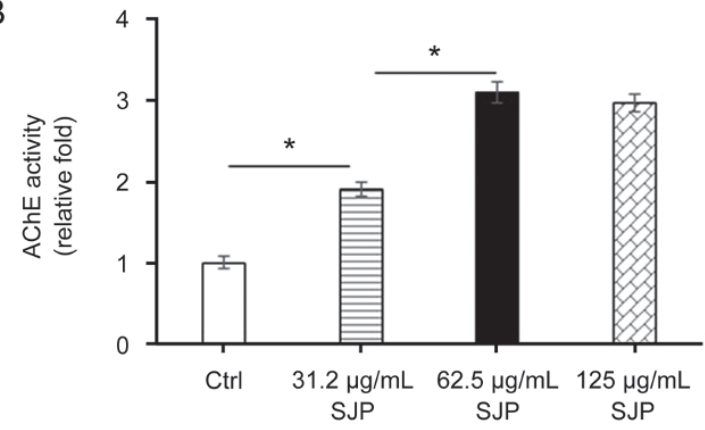

Figure 3. Quantification of acetylcholinesterase (AChE) activity of purified exosomes. (A) AChE activity of exosomes from Ctrl, SJP, TMP and BOR treated C-MSCs ( ${ }^{*} P<0.05$ vs Ctrl, TMP \& BOR, $n=6$; ${ }^{~} P<0.05$ vs Ctrl, SJP, BOR, $n=6$ ); (B) Dose effect of SJP on the AChE activity of exosomes $\left({ }^{*} P<0.05, n=3\right)$; (C) Time effect of SJP on AChE activity of C-MSC-derived exosomes $\left({ }^{*} P<0.05, n=3\right)$.

in exosomes, and AChE activity was used for exosome quantification ${ }^{[25]}$. We measured AChE activity in exosome fractions obtained from Ctrl, SJP, TMP, and BOR treated C-MSCs. As shown in Figure 3A, both SJP and TMP treatment significantly increased AChE activity compared to Ctrl treatment. Notably, SJP treatment had the highest AChE activity compared to TMP and BOR treated groups, suggesting that SJP treatment stimulates exosome secretion from C-MSCs and that there is a synergistic effect with a combination of herbs (Fangii) in traditional Chinese medicine. We further measured the dose-response and time-course of SJP on exosome secretion from C-MSCs. As shown in Figure 3B, SJP-mediated exosome release shows significant effects at $31.2 \mu \mathrm{g} / \mathrm{mL}$ and peaked at $62.5 \mu \mathrm{g} / \mathrm{mL}$. Next, we studied the time-course of SJP $(62.5 \mu \mathrm{g} / \mathrm{mL})$ on exosome release from C-MSCs and observed that SJP-stimulated exosome release peaks at $48 \mathrm{~h}$ (Figure 3C).

\section{Effect of SJP on stimulating exosome secretion from C-MSCs is nSMase2 independent}

Previous reports have shown that nSMase2 signaling commonly controls exosome secretion ${ }^{[26]}$. To identify the mechanism of SJP in stimulating the secretion in C-MSCs, we measured the mRNA expression level and protein abundance of nSMase2 in Ctrl, SJP, TMP and BOR treated C-MSCs.
A

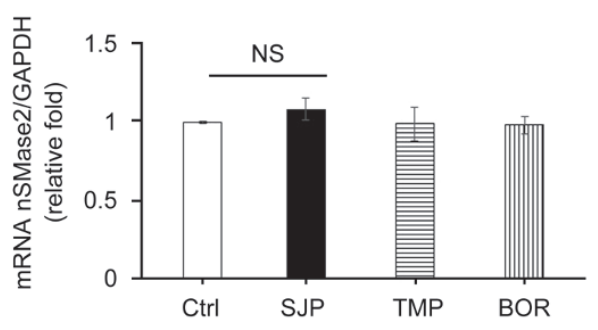

C

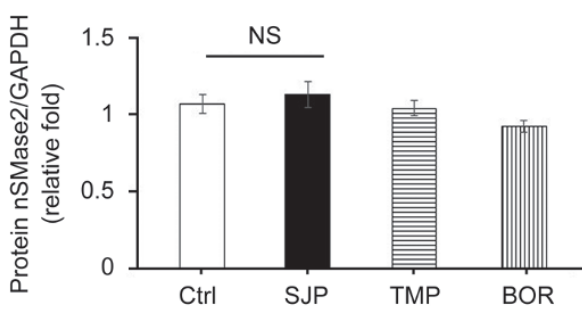

B

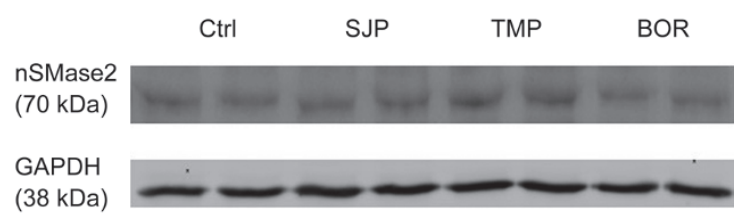

Figure 4. Identification of nSMase 2 expression in Ctrl, SJP, TMP and BOR treated C-MSCs. (A) Real-time PCR results show nSMase2 mRNA level in Ctrl, SJP, TMP and BOR treated C-MSCs [no significance (NS), $n=3$ ]; (B) Western blot results show nSMase 2 protein abundance in Ctrl, SJP, TMP and BOR treated C-MSCs; (C) Densitometric arbitrary units were normalized to GAPDH abundance and are expressed as the mean \pm SEM (NS, $n=3$ ). 
A

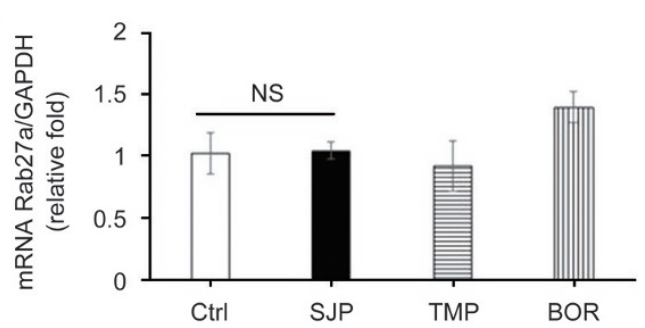

C

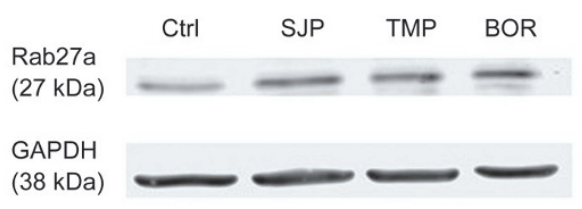

E

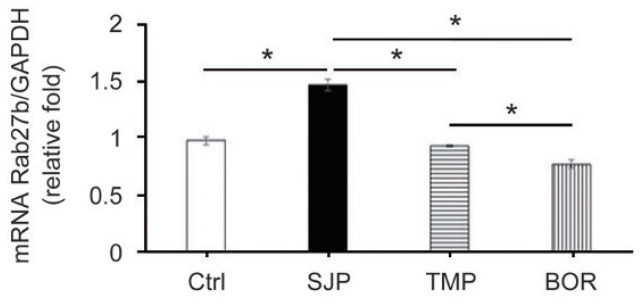

G

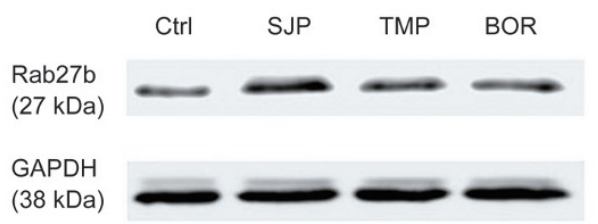

I

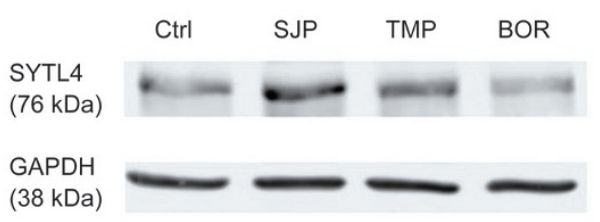

B

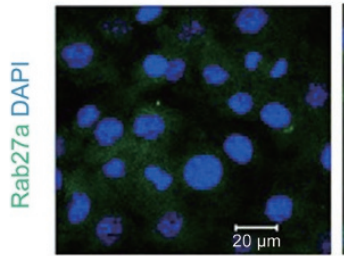

Ctrl

D

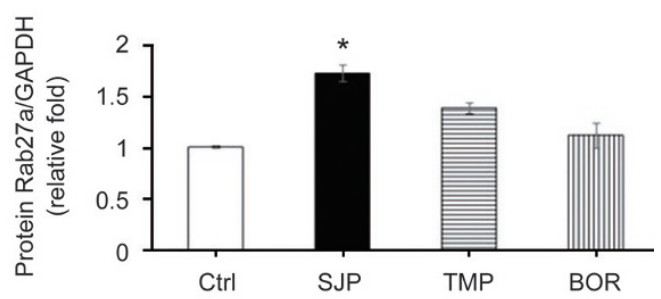

F

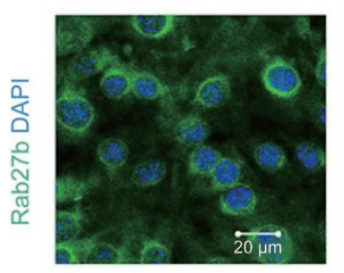

Ctrl

$\mathrm{H}$

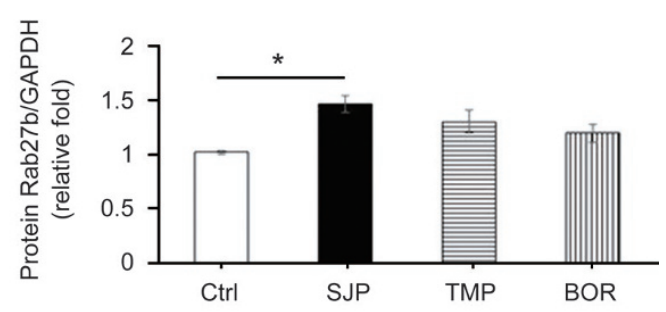

$\mathrm{J}$

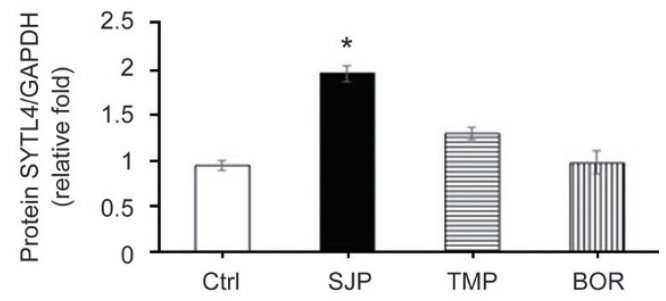

Figure 5. Identification of Rab27a and Rab27b expression in Ctrl, SJP, TMP and BOR treated C-MSCs. (A) Real-time PCR results show Rab27a mRNA expression in Ctrl, SJP, TMP and BOR treated C-MSCs (NS, $n=3$ ); (B) Staining of Rab27a in Ctrl and SJP treated C-MSCs; (C, D) Western blot results show protein level of Rab27a in Ctrl, SJP, TMP and BOR treated C-MSCs. Densitometric arbitrary units were normalized to GAPDH abundance and are expressed as the mean \pm SEM ( ${ }^{*} P<0.05$ vs Ctrl, TMP \& BOR, $n=5$ ); (E) Real-time PCR results show Rab27b mRNA expression in Ctrl, SJP, TMP and BOR treated C-MSCs ( $\left.{ }^{*} P<0.05, n=3\right)$; (F) Staining of Rab27b in Ctrl and SJP treated C-MSCs; (G, H) Western blot results show protein level of Rab27b in Ctrl, SJP, TMP and BOR treated C-MSCs. Densitometric arbitrary units were normalized to GAPDH abundance and are expressed as the mean \pm SEM $\left({ }^{*} P<0.05\right.$, $n=5)$; (I, J) Western blot results show protein level of SYTL4 in Ctrl, SJP, TMP and BOR treated C-MSCs. Densitometric arbitrary units were normalized to GAPDH abundance and are expressed as the mean \pm SEM ( ${ }^{*} P<0.05$ vs Ctrl, TMP \& BOR, $\left.n=3\right)$. 
However, qRT-PCR and Western blot experiments showed that there was no significant difference among the four groups, suggesting that SJP stimulates exosome release via an nSMase2-independent pathway (Figure 4A-4C).

\section{SJP treatment regulates small GTPase expression in C-MSCs}

Rab27, a small GTPase in the Rab family, controls exosome secretion. The small GTPases Rab27a and Rab27b control membrane trafficking and microvesicle transport pathways, in particular the secretion of exosomes at different steps ${ }^{[27]}$. We first measured the mRNA and protein levels of Rab27a among Ctrl, SJP, TMP and BOR treated C-MSC groups and found that SJP treatment does not increase the mRNA level of Rab27a compared with Ctrl. However, SJP, TMP and BOR treatment significantly increased the protein abundance of Rab27a in C-MSCs, suggesting a complicated, non-transcriptional regulation of Rab27a (Figure 5A-5D). Next, we assayed the mRNA expression level and protein abundance of Rab27b among Ctrl, SJP, TMP and BOR treated C-MSCs. We found that SJP treatment significantly increases both Rab27b mRNA and protein in treated C-MSCs compared with control (Figure 5E-5H). Silencing the Rab27 effector, SYTL4, inhibits exosome secretion and phenocopies silencing Rab27a ${ }^{[26]}$. We assayed SYTL4 protein abundance among Ctrl, SJP, TMP and BOR treated C-MSCs and found that SJP treatment significantly increased SYTL4 protein compared with the control (Figure 5I, 5J). Finally, we assayed Tsg101 and CD63 protein abundance among Ctrl, SJP, TMP and BOR treated C-MSCs and also observed that SJP treatment significantly increases Tsg101 and
CD63 protein compared with the control (Figure 6).

The role of Rab27a and Rab27b on SJP-mediated exosome secretion To determine whether increased Rab27a and Rab27b were responsible for increased exosome release post-SJP treatment in C-MSC, we knocked down Rab27a or Rab27b expression in C-MSCs by transfection with si-Rab27a or si-Rab27b. qRTPCR showed that Rab27a-siRNA knocked down Rab27a expression approximately 80\% (Figure 7A), and Rab27bsiRNA knocked down Rab27b expression approximately 64\% (Figure 7B). We observed that the SJP-induced increased exosome secretion was slightly inhibited by Rab27a knockdown (Figure 7C). However, Rab27b knockdown almost abolished the SJP-induced increase of exosome secretion in C-MSCs (Figure 7D). Electroporation by the Neon Transfection System may interrupt SJP-mediated exosome secretion as SJP treatment only increased exosome release in the NT-siRNA group by approximately 1.8 -fold, which is far less than its effect on un-transfected C-MSC. Collectively, these observations indicate that the exosome secretion of C-MSCs is increased by SJP and that the mechanism of increased exosome release is dependent on an SJP-mediated increase of Rab27b expression.

\section{Discussion}

This is the first observation that the Suxiao Jiuxin pill can stimulate exosome secretion from cardiac mesenchymal stem cells in vitro. In our experiments, we found that SJP treatment stimulates exosome secretion from C-MSCs with a significant increase in Rab27a, SYTL4, and Rab27b protein, but

A

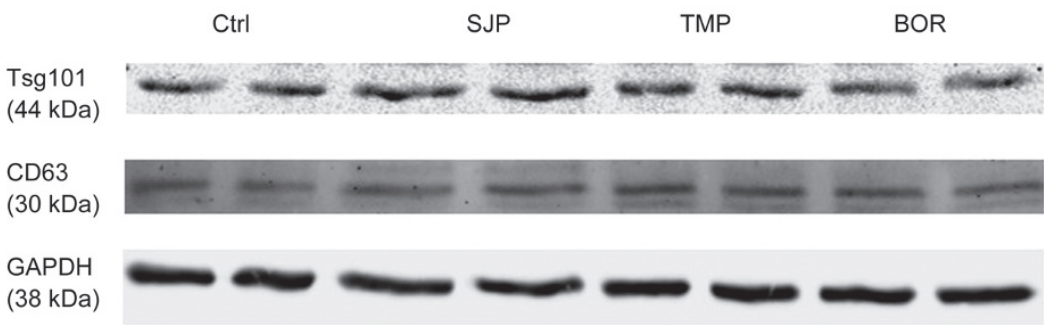

B

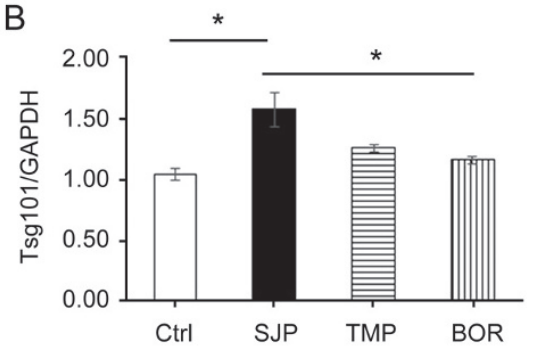

C

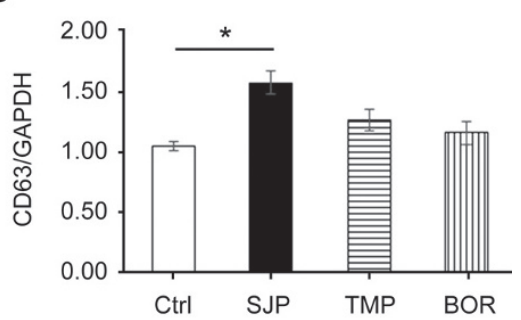

Figure 6. Identification of Tsg101 and CD63 expression in Ctrl, SJP, TMP and BOR treated C-MSCs. (A, B) Western blot result shows protein level of Tsg101 in Ctrl, SJP, TMP and BOR treated C-MSCs. Densitometric arbitrary units were normalized to GAPDH abundance and are expressed as the mean \pm SEM $\left({ }^{*} P<0.05, n=4\right)$; (A, C) Western blot result shows protein level of CD63 in Ctrl, SJP, TMP and BOR treated C-MSC. Densitometric arbitrary units were normalized to GAPDH abundance and are expressed as the mean \pm SEM $\left({ }^{*} P<0.05, n=3\right)$. 
A

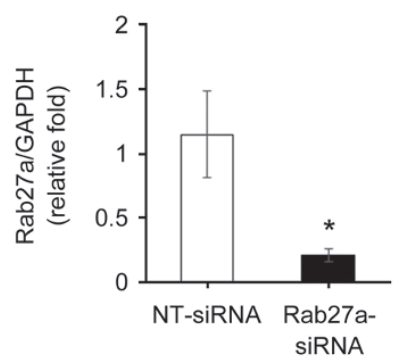

C

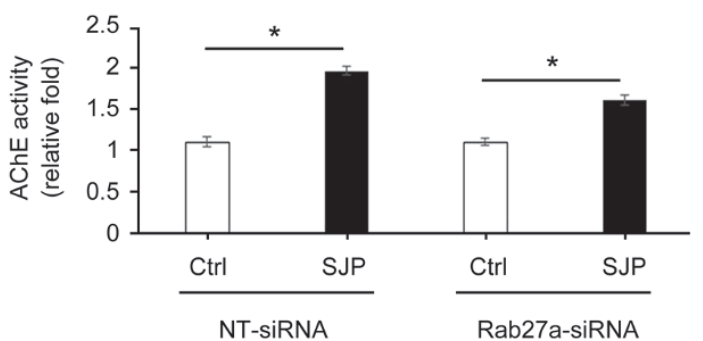

B

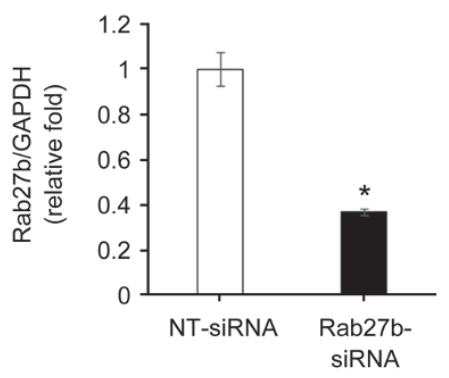

D

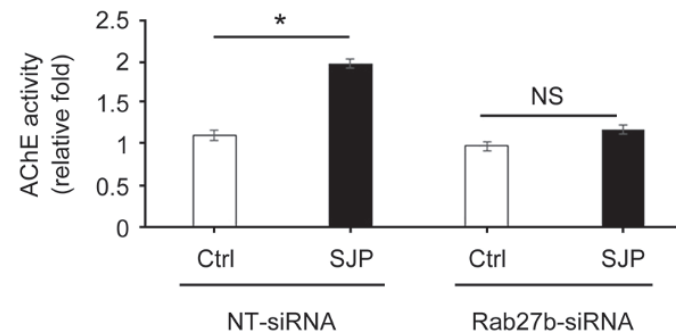

Figure 7. Knockdown of Rab27a or Rab27b inhibits SJP-mediated exosome release in C-MSCs. (A, B) qRT-PCR quantification of Rab27a and Rab27b mRNA levels (normalized to GADPH levels) in C-MSCs transfected with Rab27a siRNA, Rab27b siRNA, or non-targeting (NT) siRNA ( $\left.{ }^{*} P<0.05, n=3\right)$; (C, D) Quantification of AChE activity of exosomes from Ctrl or SJP treated C-MSCs with NT-siRNA, Rab27a-siRNA or Rab27b-siRNA transfection ( ${ }^{*} P<0.05, n=3$ ).

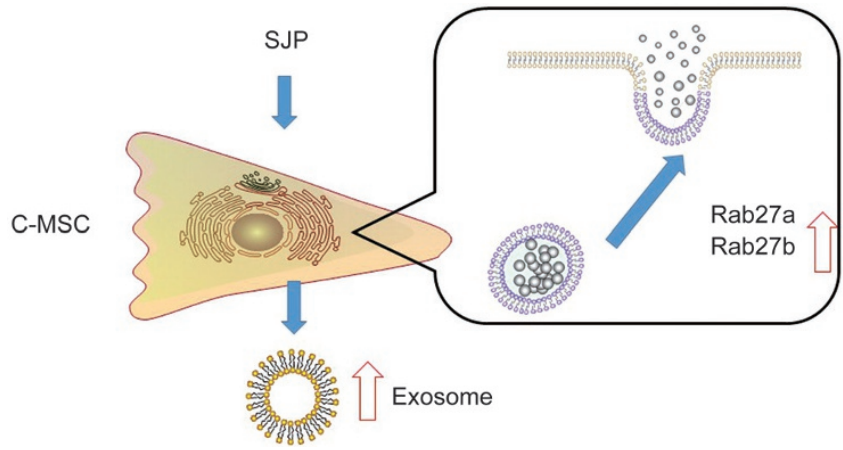

Figure 8. Schematic overview of the activation of exosome secretion signaling after SJP treatment. SJP treatment leads to upregulation of Rab27 expression and exosome secretion in C-MSCs.

not nSMase2, in the corresponding C-MSCs compared to Ctrl treatment. Moreover, the SJP-induced increase of C-MSCexosome release can be abolished by Rab27b knockdown. Collectively, these observations indicate that SJP stimulates exosome release via small GTPases in an nSMase2-independent pathway.

We observed that there is difference in exosome quantification between the AChE activity assay and nanoparticle tracking analysis (NTA). The NTA assay showed a 2.5-fold increase of exosome release by SJP treatment $\left(1.1 \mathrm{E}+10\right.$ particles $/ \mathrm{cm}^{3}$ in SJP vs 4.4E+9 particles $/ \mathrm{cm}^{3}$ in Ctrl) while the AChE assay show an approximate 3-5-fold increase by SJP treatment compared with control treatment. Because exosomes are sticky and sometimes accumulate to form grape-like clusters under electron microscopy (Figure 2A), this phenomenon might help us explain why the value of NTA quantification is lower than
AChE activity.

In this study, we found that SJP treatment does not increase mRNA levels of Rab27a expression but significantly increased Rab27a protein abundance, which might be due to posttranscriptional regulation. Post-transcriptional gene expression occurs on at least three levels, including (1) RNA posttranscriptional modification, which is a dynamic and tissue specific deposition of $\mathrm{m} 6 \mathrm{~A}, \psi$, and $\mathrm{m} 5 \mathrm{C}$ in RNA, which therefore regulates protein synthesis so cells adapt to environment stress ${ }^{[28]}$; (2) mRNA degradation by microRNAs, which target the $3^{\prime}$ untranslated region (3'UTR) to regulate mRNA degradation and change translational rates ${ }^{[29-31]}$; and (3) regulation of protein stability via ubiquitination and proteasome degradation. Post-transcriptional regulation is also observed in hypoxia preconditioning, which increases the protein level of hypoxia-inducible factor $1 a(H I F-1 \alpha)$ expression via stabilizing HIF-1a protein by inhibiting ubiquitin-proteasome pathwaymediated rapid degradation of the oxygen-dependent degradation (ODD) domain in HIF-1a ${ }^{[17,32]}$. However, hypoxia preconditioning reduces mRNA levels of HIF-1a via decreasing HIF-1a mRNA stability by inducing an increase in the quantity of antisense HIF-1 ${ }^{[33]}$.

Our findings demonstrated that SJP treatment increases exosome secretion from C-MSCs with Rab27b mRNA and protein expression. We found that SJP treatment shows significantly higher exosome secretion compared to TMP and BOR in C-MSCs, suggesting a synergistic effect in enhancing the exosome secretion pathway by multiple-component herbal combination, which is consistent with the concept that multiherb prescription might have synergistic effects via underlying interactive effects ${ }^{[34]}$. SJP-mediated Rab27b regulation occurs at both transcriptional and post-transcriptional levels, and SJP 
is not a single component. To determine the initiating event triggering Rab27b expression in C-MSCs, we can use a new high throughput ribosome profiling approach called translating ribosome affinity purification (TRAP) followed by RNA sequencing technology (TRAP-SEQ) ${ }^{[35]}$ to differentiate translational or post-translational regulation of Rab27b in C-MSCs. Briefly, we will transfect C-MSCs with the RPL10a ribosomal fusion protein, a core ribosomal protein, which enables affinity purification of $80 S$ ribosome translated specific mRNA genome-wide $^{[36]}$. TRAP-SEQ permits us to conduct comprehensive studies of translated mRNAs in C-MSCs in response to SJP treatment. Next, to identify transcriptional regulation of Rab27b expression by transcription factors (TFs), we performed bioinformatics analysis of both enhancer and promoter regions of Rab27b gene and found that (1) the promoter region has binding sites for p53, Gfi-1, POU2F1a, POU2F1, Ik-3, POU6F1(c2), Oct-B1, oct-B2, and oct-B3 using the DECipherment Of DNA Elements (DECODE) assay; and (2) the distal enhancer region has $28 \mathrm{TF}$ binding sites, including HDAC1, LEF1, PKNOX1, ATF1, MAX, CEBPG, FOSL1, TCF12, ZNF766, CTBP1, GATA2, NCOR1, POLR2A, TRIM24, RCOR1, CREM, SMARCE1, RNF2, SOX6, DPF2, NR2F2, ZNF592, GABPA, JUND, HDAC2, SMARCA4, HMBOX1, and HNF4A by Encyclopedia of DNA Elements (ENCODE) analyses.

Because SJP treatment can upregulate exosome signaling and increase exosome release, SJP has the therapeutic potential to promote endogenous cardiac protection via increased exosome release. SJP can regulate critical elements of exosome signaling via post-transcriptional processes and therefore might increase protective exosome release compared to de novo exosome synthesis. There is a small amount of C-MSCs in normal hearts, and therefore, the cardioprotective effect of SJP cannot be explained by stimulation of exosome secretion from C-MSCs. As the major mechanism of stem cell therapy is by paracrine effects from donor cells, we might use SJP to precondition C-MSCs to promote their paracrine effects to enhance heart repair.

In summary, we found that the SJP treatment can stimulate exosome secretion in C-MSCs via GTPase regulation (Figure 8). This finding broadens our current understanding of the function and mechanism of SJP in treating ischemic heart disease. Our findings also highlight, for the first time, the important effect of SJP on regulating exosome release from resident C-MSCs.

\section{Acknowledgements}

This study was supported by the National Natural Science Foundation of China (№ 81403352, 81573647 to Xiaolong WANG and Xiao-fen RUAN, and 81528002 to Yaoliang TANG), the Shanghai Key Laboratory of Traditional Chinese Clinical medicine (№ 14DZ2273200). This study was also partially supported by Tianjin Zhongxin Pharmaceutical Group Co, Ltd (Tianjin, China). Il-man KIM, Neal WEINTRAUB, and Yaoliang TANG were partially supported by the American Heart Association: GRNT31430008, NIH-AR070029, NIHHL086555, NIH-HL134354, and NIH-HL12425.

\section{Author contribution}

Xiao-long WANG and Yaoliang TANG designed the study; Xiao-fen RUAN, Cheng-wei JU, Yan SHEN, and Yu-tao LIU collected the data; Xiao-fen RUAN and Xiao-long WANG analysed the data; Xiao-fen RUAN, Xiao-long WANG, and Yaoliang TANG interpreted the data; Il-man KIM, Hong YU, Neal WEINTRAUB, and Yaoliang TANG wrote the manuscript.

\section{References}

1 Lu Z, Zhang Y, Zhuang P, Zhang J, Zhou H, Zhang M, et al. Protective effect of Suxiao jiuxin pill, a traditional Chinese medicine, against acute myocardial ischemia in dogs. BMC Complement Altern Med 2015; 15: 373.

2 Duan X, Zhou L, Wu T, Liu G, Qiao J, Wei J, et al. Chinese herbal medicine suxiao jiuxin wan for angina pectoris. Cochrane Database Syst Rev 2008; (1): Cd004473.

3 Jia YL, Huang FY, Zhang SK. Assessment of the quality of randomized controlled trials in treating coronary heart disease by Chinese patent medicine. Zhongguo Zhong Xi Yi Jie He Za Zhi 2012; 32: 560-8.

4 Wang XL, Liu YM, Zhu GJ. Effects of suxiao jiuxin pill on patients with acute coronary syndrome undergoing early percutaneous coronary intervention. Zhongguo Zhong Xi Yi Jie He Za Zhi 2012; 32: 1483-7.

5 Hong YL, Jin YQ, Yao YX, Lin MY, Wei BP, Jiang WD, et al. Assay of Tetramethylpyrazine in Szechwan Lovage Rhizome and Cnidium Rhizome by HPLC-DAD-MS. Zhong Yao Cai 2015; 38: 36-40.

6 CS Li, Qu ZQ, Wang SS, Hao XW, Zhang XQ, Guan J, et al. Effects of suxiao jiuxin pill (see test) on oxidative stress and inflammatory response in rats with experimental atherosclerosis. J Tradit Chin Med 2011; 31: 107-11.

7 Bai XY, Zhang P, Yang Q, Liu XC, Wang J, Tong YL, et al. Suxiao jiuxin pill induces potent relaxation and inhibition on contraction in human artery and the mechanism. Evid Based Complement Alternat Med 2014; 2014: 956924.

8 Tang YL, Shen L, Qian K, Phillips MI. A novel two-step procedure to expand cardiac Sca-1+ cells clonally. Biochem Biophys Res Commun 2007; 359: 877-83.

9 Tang YL, Zhao Q, Qin X, Shen L, Cheng L, Ge J, et al. Paracrine action enhances the effects of autologous mesenchymal stem cell transplantation on vascular regeneration in rat model of myocardial infarction. Ann Thorac Surg 2005; 80: 229--36.

10 Tang YL, Zhao Q, Zhang YC, Cheng L, Liu M, Shi J, et al. Autologous mesenchymal stem cell transplantation induce VEGF and neovascularization in ischemic myocardium. Regul Pept 2004; 117: 3-10.

11 Campbell CR, Berman AE, Weintraub NL, Tang YL. Electrical stimulation to optimize cardioprotective exosomes from cardiac stem cells. Med Hypotheses 2016; 88: 6-9.

12 Wang Y, Zhang L, Li Y, Chen L, Wang X, Guo W, et al. Exosomes/ microvesicles from induced pluripotent stem cells deliver cardioprotective miRNAs and prevent cardiomyocyte apoptosis in the ischemic myocardium. Int J Cardiol 2015; 192: 61-9.

13 Chen L, Wang Y, Pan Y, Zhang L, Shen C, Qin G, et al. Cardiac progenitor-derived exosomes protect ischemic myocardium from acute ischemia/reperfusion injury. Biochem Biophys Res Commun 2013; 431: 566-71.

14 Wang X, Gu H, Huang W, Peng J, Li Y, Yang L, et al. Hsp20-mediated activation of exosome biogenesis in cardiomyocytes improves cardiac function and angiogenesis in diabetic mice. Diabetes 2016; 65: 3111-28. 
15 Guo BB, Bellingham SA, Hill AF. The neutral sphingomyelinase pathway regulates packaging of the prion protein into exosomes. J Biol Chem 2015; 290: 3455-67.

16 Zheng Y, Campbell EC, Lucocq J, Riches A, Powis SJ. Monitoring the Rab27 associated exosome pathway using nanoparticle tracking analysis. Exp Cell Res 2013; 319: 1706-13.

17 Tang YL, Zhu W, Cheng M, Chen L, Zhang J, Sun T, et al. Hypoxic preconditioning enhances the benefit of cardiac progenitor cell therapy for treatment of myocardial infarction by inducing CXCR4 expression. Circ Res 2009; 104: 1209-16.

18 National Pharmacopoeia Committee. Chinese Pharmacopoeia 2015.

19 Guo J, Meng H, Wang L, Zhang L, Hang X, Xu R. Effect of blood drug level change of Borneol in Suxiaojiuxin pill on patients with myocardial ischemia. Modern J Integr Tradit Chin Western Med 2004; 13: 2387 402.

20 Yu L, Li M, She T, Han L. Effects of Tetramethylpyrazine on Ang IIinduced cardiomyocyte hypertrophy and the underlying mechanisms. Chin J Exp Tradit Med Form 2013; 19: 154-7.

21 Lee C, Mitsialis SA, Aslam M, Vitali SH, Vergadi E, Konstantinou G, et al. Exosomes mediate the cytoprotective action of mesenchymal stromal cells on hypoxia-induced pulmonary hypertension. Circulation 2012; 126: 2601-11.

22 Helwa I, Cai J, Drewry MD, Zimmerman A, Dinkins MB, Khaled ML, et al. A comparative study of serum exosome isolation using differential ultracentrifugation and three commercial reagents. PLoS One 2017; 12: e0170628.

23 Essandoh K, Yang L, Wang X, Huang W, Qin D, Hao J, et al. Blockade of exosome generation with GW4869 dampens the sepsis-induced inflammation and cardiac dysfunction. Biochim Biophys Acta 2015; 1852: 2362-71.

24 Nery AA, Nascimento IC, Glaser T, Bassaneze V, Krieger JE, Ulrich H. Human mesenchymal stem cells: from immunophenotyping by flow cytometry to clinical applications. Cytometry A 2013; 83: 48-61.

25 Lv LH, Wan YL, Lin Y, Zhang W, Yang M, Li GL, et al. Anticancer drugs cause release of exosomes with heat shock proteins from human hepatocellular carcinoma cells that elicit effective natural killer cell antitumor responses in vitro. J Biol Chem 2012; 287: 15874-85.

26 Ostrowski M, Carmo NB, Krumeich S, Fanget I, Raposo G, Savina A, et al. Rab27a and Rab27b control different steps of the exosome secretion pathway. Nat Cell Biol 2010; 12: 19-30; sup pp 1-13.

27 Chen TC, Hsieh CH, Sarnow P. Supporting role for GTPase Rab27a in Hepatitis C virus RNA replication through a novel miR-122-mediated effect. PLoS Pathog 2015; 11: e1005116.

28 Frye M, Blanco S. Post-transcriptional modifications in development and stem cells. Development 2016; 143: 3871-81.

29 Vlasova-St Louis I, Bohjanen PR. Post-transcriptional regulation of cytokine and growth factor signaling in cancer. Cytokine Growth Factor Rev 2017; 33: 83-93.

30 Zhang L, Zhou M, Qin G, Weintraub NL, Tang Y. MiR-92a regulates viability and angiogenesis of endothelial cells under oxidative stress. Biochem Biophys Res Commun 2014; 446: 952-8.

31 Zhang L, Zhou M, Wang Y, Huang W, Qin G, Weintraub NL, et al. miR92a inhibits vascular smooth muscle cell apoptosis: role of the MKK4JNK pathway. Apoptosis 2014; 19: 975-83.

32 Tang YL, Tang Y, Zhang YC, Agarwal A, Kasahara H, Qian K, et al. A hypoxia-inducible vigilant vector system for activating therapeutic genes in ischemia. Gene Ther 2005; 12: 1163-70.

33 Uchida T, Rossignol F, Matthay MA, Mounier R, Couette S, Clottes E, et al. Prolonged hypoxia differentially regulates hypoxia-inducible factor (HIF)-1alpha and HIF-2alpha expression in lung epithelial cells: implication of natural antisense HIF-1alpha. J Biol Chem 2004; 279: 14871-8.

34 Che CT, Wang ZJ, Chow MS, Lam CW. Herb-herb combination for therapeutic enhancement and advancement: theory, practice and future perspectives. Molecules 2013; 18: 5125-41.

35 Reynoso MA, Juntawong P, Lancia M, Blanco FA, Bailey-Serres J, Zanetti ME. Translating Ribosome Affinity Purification (TRAP) followed by RNA sequencing technology (TRAP-SEQ) for quantitative assessment of plant translatomes. Methods Mol Biol 2015; 1284: 185-207.

36 Shi Z, Fujii K, Kovary KM, Genuth NR, Rost HL, Teruel MN, et al. Heterogeneous ribosomes preferentially translate distinct subpools of mRNAs genome-wide. Mol Cell 2017; 67: 71-83.e7. 